\title{
Propriétés physiques et hydrauliques de sédiments marins en vue de réutilisation en remblais
}

\author{
Daniel LEVACHER ${ }^{1}$, Martin SANCHEZ ${ }^{2}$, Zhibo DUAN ${ }^{1}$, \\ Cécile LE GUERN ${ }^{3}$
}

${ }^{1}$ Université de Caen, Faculté des Sciences, UMR 6143 CNRS - M2C,

Esplanade de la Paix, 14032 Caen, France

daniel.levacher@unicaen.fr; zhibo.duan@unicaen.fr.

${ }^{2}$ Université de Nantes, UMR 6112 CNRS - Planétologie et Géodynamique,

2 rue de la Houssinière, 44322 Nantes, France

martin.sanchez@univ-nantes.fr

${ }^{3}$ BRGM Nantes, 1 Rue des Saumonières, 44300 Nantes, France

cecile.leguern@brgm.fr

\section{Résumé :}

Toute valorisation de sédiments fins marins de dragage impose une méthodologie de caractérisation (physique, hydraulique et mécanique). Celle-ci a été proposée pour le cas de sédiments marins de la Manche ( 2 sédiments) et de la Méditerranée (6 sédiments). Après en avoir défini les propriétés physiques, des essais de décantation à la colonne pour différentes concentrations ont été effectués sur ces 8 sédiments. L'analyse des résultats a tout d'abord porté sur l'étude des courbes de tassement à la colonne et sur l'établissement de loi en fonction de la concentration. Sont alors déduites, les relations entre la perméabilité et l'indice des vides. Pour simuler le tassement de ces mêmes sédiments sous surcharge, des essais œdométriques ont été réalisés. L'analyse a conduit à l'obtention des paramètres de consolidation.

\section{Mots-clés :}

Sédiment de dragage - Propriétés physiques - Sédimentation - Consolidation Tassement - Perméabilité

\begin{abstract}
:
To reuse dredged sludge for earth structures such as unconfined or non embankments, a methodology is proposed to characterize marine sediments. This concerns physical, hydraulic and consolidation properties which constitutes a
\end{abstract}


basis for the design of any embankment with sediments. A series of 8 marine sediments coming from the French Channel coast (2) and Mediterranean Sea (6) has been studied using different sedimentation, oedometric and creep testing. All the results are analyzed and presented in this paper. The main data are relative to the grain size distribution and Atterberg limits and relationships between permeability and void ratio or concentration. Finally some consolidation data are summarized in comparison with compressible soils.

\section{Keywords :}

Dredged sludge - Physical properties - Sedimentation - Consolidation Settlement - Permeability

\section{Introduction}

Ports, fleuves, barrages fournissent inlassablement des volumes considérables (des millions de $\mathrm{m}^{3}$ ) de sédiments fins de dragage de taille inférieure à $80 \mu \mathrm{m}$. L'élimination de ces matériaux pose problème dans un premier temps. Il y a les solutions traditionnelles pour y répondre : le clapage en mer (Port du Havre) avec l'obligation de satisfaire les règles relatives à l'immersion de sédiments marins du décret du 10 août 2000 (ARRÊTÉ, 2000), la mise en dépôts à terre dans des zones tampons disposant de gros volumes comme les ballastières (Port de Rouen), le stockage provisoire pour de petits volumes (en casiers, sur des sites en carrière, à proximité de barrages), le réemploi du fait de caractéristiques spécifiques des matériaux fins (amendement ou épandage agricole, (LE GUERN et al., 2004)). Dès lors qu'il s'agit de matériaux fins contaminés, ces méthodes traditionnelles ne s'appliquent plus, ce sont des déchets à traiter et ce, selon la nature même des contaminants. Ces derniers représentent des volumes réduits (des milliers de $\mathrm{m}^{3}$ ).

Valoriser les sédiments marins constitue donc un enjeu important dans la gestion de ceux-ci. Les récents travaux de recherche à ce sujet étaient très orientés «matériaux routiers et matériaux de construction». Les objectifs visés étaient ambitieux connaissant toutes les difficultés à stabiliser et/ou solidifier les sédiments marins ou fines de barrages et les échecs quant à l'application sur site. Il est important de citer trois types de travaux réalisés sur des sédiments marins et fines de barrage, travaux marquants par le fait que pour les études menées sur les sédiments, l'aspect économique a été considéré (COLIN, 2003 ; LEMEE, 2006) et que pour les boues de barrage (SEMCHA, 2006), un matériau a été mis au point.

Toute valorisation de matériaux fins dragués peut être hiérarchisée (LEVACHER et al., 2007a) selon les différents objectifs à atteindre, en accord avec le tableau suivant. 
Tableau 1. Domaines possibles de valorisation

\begin{tabular}{|l|l|}
\hline Objectif & Application et domaine concerne \\
\hline $\begin{array}{l}\text { 1-Pelletabilité du produit en cours de } \\
\text { stabilisation/solidification. }\end{array}$ & $\begin{array}{l}\text { Transport et mise en décharge (matériau fin } \\
\text { contaminé), mise en dépôt pour stockage. }\end{array}$ \\
\hline $\begin{array}{l}\text { 2-Mise en dépôt : remplissage de carrières, de } \\
\text { cavités souterraines. }\end{array}$ & Matériau de remplissage, de comblement. \\
\hline 3-Réalisation de remblai sans surcharge. & $\begin{array}{l}\text { Consolidation sous poids propre, zones } \\
\text { aménagées. }\end{array}$ \\
\hline 4-Remblai avec surcharge. & $\begin{array}{l}\text { Matériau de terre-pleins et de plateformes } \\
\text { industriels. }\end{array}$ \\
\hline 5-Valorisation en technique routière. & Matériau de sous-couches routières. \\
\hline 6-Valorisation en matériaux élaborés. & Matériaux de construction (briques, tuiles). \\
\hline
\end{tabular}

Toute valorisation relative à la réutilisation des sédiments marins en remblai impose une caractérisation adéquate. Elle concerne précisément l'étude des propriétés essentielles que sont les propriétés physiques, hydrauliques et de consolidation. Ce sont celles-ci qui sont présentées par la suite pour une série de 8 sédiments.

\section{Organisation, moyens et méthodes d'une caractérisation}

\subsection{Prétraitements appliqués aux sédiments naturels}

Les sédiments à valoriser ont subi des opérations de tri et de séchage partiel. Le tri dimensionnel vise d'une part, à éliminer les macro déchets, les blocs, les cailloux de manière à ce que le diamètre maximal n'excède pas 100, 80 voire $20 \mathrm{~mm}$ à l'aide d'unités de dégrillage ou de cribles, et d'autre part à isoler par criblage à sec ou éventuellement humide, les sables grossiers et graviers dont le diamètre minimal est à fixer notamment pour envisager des traitements de solidification à l'aide de liants hydrauliques et additifs. Il est souvent situé à 2 ou $4 \mathrm{~mm}$. En effet, bon nombre de travaux de recherche ont montré que la fraction sableuse fine joue un rôle dans la résistance mécanique de sédiments fins traités aux liants hydrauliques (COLIN, 2003 ; DUBOIS, 2005 ; LEVACHER et al., 2007b). Les sédiments recueillis correspondent donc à des classes de $0 / 2$ ou 0/4 mm. Poursuivre le tri s'impose parfois dans le cas de sédiments contaminés afin de concentrer les contaminants sur la partie fine inférieure à 80 ou $63 \mu \mathrm{m}$; cette séparation se fait par hydrocyclonage. Pour abaisser la teneur en eau, un séchage partiel peut être effectué sur les sédiments triés au niveau des fines ou sur la classe granulaire $0 / 2 \mathrm{~mm}$ ou plus, soit naturellement (lit de séchage) soit par pressage (filtre-presse). 


\subsection{Caractérisation des sédiments naturels}

Les classes granulaires des sédiments naturels faisant l'objet de valorisation quelle qu'en soit la finalité vont de 0 à $5 \mathrm{~mm}$ comprenant les fines et les sables. Au-delà de cette taille maximale, les matériaux grossiers contenus dans les sédiments peuvent être réutilisés comme granulats s'il s'agit des cailloux ou blocs. La classe granulaire des sédiments étudiés pour une réutilisation en remblai est de $0 / 2 \mathrm{~mm}$. La caractérisation de sédiments marins pour toute valorisation et en particulier une réutilisation en remblai, doit comporter la détermination des propriétés suivantes :

- Propriétés physiques : répartition dimensionnelle, répartition des éléments fins, répartition des sables fins, indice de plasticité et limites d'Atterberg.

- Propriétés chimiques : teneur en matières organiques, teneur en carbonates.

- Propriétés environnementales : teneur en contaminants (cas de sédiments pollués).

- Paramètres de décantation : relation k-C (perméabilité-concentration), loi de décantation.

- Paramètres de consolidation : relation $\mathrm{k}-\mathrm{C}$, coefficients de consolidation et de fluage, indice de compression et de gonflement.

- Paramètres de résistance : relation $\mathrm{W}-\mathrm{Cu}$ (teneur en eau $\mathrm{W}$ - cohésion non drainée) ou relation $\mathrm{C}-\mathrm{Cu}$.

Cette caractérisation apporte tous les éléments nécessaires à une valorisation des sédiments en remblai sans traitement (remblai confiné ou non) et permet d'envisager un traitement de stabilisation et/ou de solidification avec additifs. Pour les autres filières avec traitement, de stabilisation et/ou solidification, un second volet d'études s'impose qui comprend différentes phases de validation d'indicateurs comportementaux : compactage, mécaniques et environnementaux et, économiques. Pour nos 8 sédiments, nous nous sommes focalisés sur les propriétés physiques et hydrauliques et ce pour une application en remblai.

\subsection{Apport d'une caractérisation chimique et minérale}

L'applicabilité de techniques particulières de traitement aux sédiments marins notamment celles de stabilisation/solidification qui utilisent des réactifs chimiques requiert une caractérisation chimique et minéralogique. En effet la caractérisation des principales phases cristallisées majeures peut se faire par diffractométrie aux rayons X. De la prépondérance de minéraux siliceux et/ou carbonatés dans un sédiment dépend le choix du produit chimique (LEMEE, 2006, LIANG, 2008).

La connaissance des compositions minéralogiques permet d'envisager d'autres valorisations comme l'incorporation des matières sèches calcinées pour élaborer 
une pouzzolane de synthèse (SEMCHA, 2006) selon les teneurs en argile et calcaire présentes au sein du sédiment.

L'analyse des espèces argileuses qui composent le cortège argileux des sédiments peut s'avérer intéressante. En effet, la présence de smectite en forte proportion par exemple dans un sédiment fluvial, voir tableau 2, peut poser des problèmes pour une réutilisation en remblai, mais aussi pour une valorisation par liants hydrauliques. En effet cette espèce argileuse est fortement sujette aux variations de l'état hydrique du milieu où elle se trouve. Qui plus est, la smectite possède une grande surface spécifique de l'ordre de $800 \mathrm{~m}^{2} / \mathrm{g}$ et joue un rôle de pralinage autour des particules minérales. En recouvrant ces dernières, elle constitue une interface entre le grain et le liant apporté ce qui confère aux hydrates une mauvaise adhérence (COLIN, 2003). Les effets de la smectite en remblai sont étudiés à travers les paramètres de consolidation et d'essais de gonflement.

Tableau 2. Quelques constituants de sédiments

\begin{tabular}{|c|c|c|c|c|c|}
\hline \multirow{2}{*}{$\begin{array}{c}\text { Sédiment } \\
\downarrow\end{array}$} & \multicolumn{5}{|c|}{ Espèces argileuses ( $\%$ de la fraction phylliteuse) } \\
\hline & Illite & Smectite & Kaolinite & Chlorite & Autres \\
\hline Calais* & 37 & 29 & 17 & 17 & - \\
\hline Toulon* & 81 & - & 18 & - & 1 \\
\hline Rouen** & 20 & 65 & 15 & - & - \\
\hline \multirow[b]{2}{*}{$\begin{array}{c}\text { Sédiment } \\
\downarrow\end{array}$} & \multicolumn{5}{|c|}{ Autres constituants } \\
\hline & Quartz (\%) & $\mathrm{CaCO}_{3}(\%)$ & $\begin{array}{c}\text { Matières } \\
\text { organiques } \\
(\%)\end{array}$ & $\begin{array}{c}\text { Fraction } \\
\text { phylliteuse } \\
(\%)\end{array}$ & $\begin{array}{c}\mathrm{NaCl} \\
(\%)\end{array}$ \\
\hline Calais & 19 & 33.9 & 3 & 20 & 6.4 \\
\hline Toulon & 15 & 17.5 & 10.4 & 40 & 6.9 \\
\hline Rouen & - & - & 7.1 & 8 & - \\
\hline
\end{tabular}

* LE GUERN et al (2004), COLIN (2003).

\section{Similitude dimensionnelle et différences des sédiments à traiter}

Une première remarque s'impose. Les sédiments faisant l'objet d'études pour un traitement ou une valorisation ont sensiblement la même classe granulaire de par les prétraitements subis. Les diamètres maximaux $\left(\mathrm{d}_{\max }\right)$ sont le plus souvent de $2 \mathrm{~mm}$. Qui plus est, dans certains travaux de recherche, ce $\mathrm{d}_{\max }$ correspond à la limite de la taille des particules que peut admettre le granulomètre laser utilisé. A titre d'exemple, sur les figures 1 et 2, LE GUERN et al. (2004) ont rassemblé une série de sédiments portuaires. On observe que du point de vue dimensionnel, les 
diamètres caractéristiques varient peu excepté dans la partie fine $\left(\mathrm{d}_{10}\right)$ mais le pourcentage des particules inférieures à $63 \mu \mathrm{m}$ est sensiblement le même.

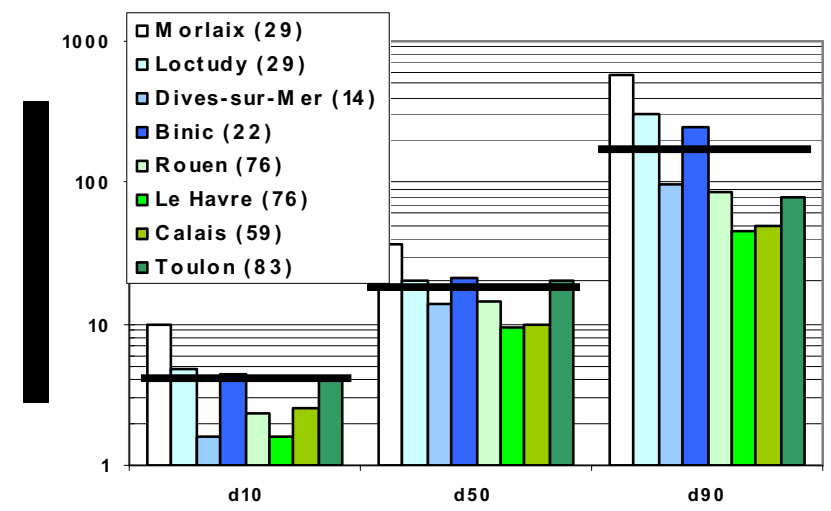

Figure 1. Diamètres caractéristiques de sédiments portuaires

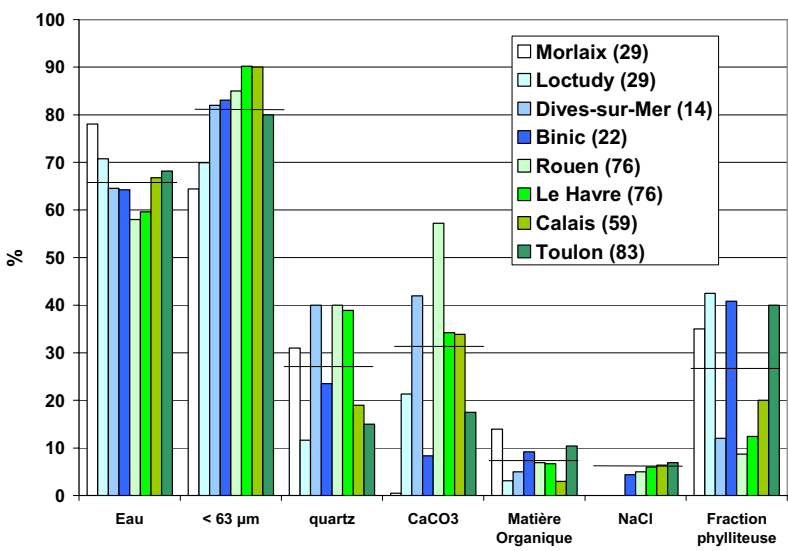

Figure 2. Constituants de sédiments portuaires

Les sédiments diffèrent les uns des autres par la nature des constituants : présence de carbonates ou de silices (intérêt pour l'utilisation de traitement chimique à base de polymères ou réactifs), présence de matières organiques (effets perturbateurs dans les stabilisations aux liants hydrauliques) et bien sûr teneurs en contaminants dans le cas de sédiments pollués.

\section{Propriétés physiques de sédiments}

Les sédiments étudiés qui ont fait l'objet d'une caractérisation minimale de base, concernent deux sédiments bruts du port de Cherbourg (réf AP et BAF respectivement pour l'avant-port et le bassin à flot)) et 6 sédiments portuaires méditerranéens provenant de l'Arsenal de Toulon (réf. ARS), de Bandol (réf. BAN), de Cannes (réf. CAN), de Sanary-Lavandou (réf. SAN), de Saint-Mandrier (réf. STM) et du Vieux Port de Marseille (réf. VPM). Cette caractérisation de base 
est physico mécanique, c'est-à-dire que les propriétés chimiques liées principalement au degré de contamination ne font pas partie de l'étude et que le comportement environnemental des sédiments étudiés n'est pas abordé, (LEVACHER et al., 2007a ; LEVACHER et al., 2007b). Cette caractérisation de base concerne donc: les propriétés physiques, à savoir : teneurs en eau et en matières organiques, limites d'Atterberg et études granulométriques. Les moyens mis en œuvre sont ceux d'essais classiques en mécanique des sols fins pour les propriétés physiques qui permettent d'aboutir à une classification utile pour les valorisations dédiées aux matériaux routiers ou en construction. L'analyse des résultats issus des essais a permis, d'une part de rassembler les diverses caractéristiques et paramètres de chaque sédiment étudié mais d'autre part, de constituer des «familles» de sédiments. Une fois connues, ces «familles» de sédiments peuvent faire l'objet de caractérisations complémentaires pour les valorisations envisagées. Il est alors considéré « la famille » et non pas pour un seul sédiment. Ceci évite d'adapter un processus de traitement pour chaque sédiment comme dans bon nombre d'études de valorisation.

Les principaux résultats concernent tout d'abord les propriétés physiques. Le mode de transport et de stockage des sédiments en bidons hermétiques s'est avéré concluant. Les teneurs en eau sont stables. Lors de manipulations, il convient d'homogénéiser le sédiment avant toute mesure de teneur en eau. Pour toute opération ou essai un tamisage à $5 \mathrm{~mm}$ a été effectué sur les sédiments bruts.

Les granulométries ont été réalisées sur huit sédiments bruts. A l'examen des fractions granulaires des fines, la fraction silteuse est la plus importante, de l'ordre de 50 à $75 \%$. Les valeurs des matières organiques effectuées sur les sédiments montrent d'une part que les valeurs sont élevées par rapport aux sédiments marins d'estuaire français et d'autre part cette matière organique se retrouve surtout sur la fraction très fine, i.e. sur les sédiments dessablés déshydratés des ports méditerranéens, comme indiqué au tableau 3. A noter que les granulométries effectuées sur ces sédiments dessablés /déshydratés notés DD montrent qu'il n'y a pas de variation de forme de la courbe granulométrique et les six sédiments sont relativement proches.

Compte tenu de ces résultats, les limites d'Atterberg réalisées sur les sédiments bruts ont confirmé selon la classification des sols fins (abaque de plasticité de Casagrande), que les sédiments étudiés étaient des limons (silts) organiques plus ou moins plastiques (figure 3). Aussi un groupe de sédiments apparaît formé des sédiments référencés ARS, BAN, CAN et SAN. 
Tableau 3. Teneurs en matières organiques des sédiments étudiés

\begin{tabular}{|c|c|c|c|}
\hline $\begin{array}{c}\text { Références } \\
\text { des sédiments }\end{array}$ & $\begin{array}{c}\text { Teneur en Matières Organiques } \\
(\%)\end{array}$ & $\begin{array}{c}\text { Références } \\
\text { des sédiments }\end{array}$ & $\begin{array}{c}\text { Teneur en Matières Organiques } \\
(\%)\end{array}$ \\
\hline ARS/BRUT & 5.1 & ARS/DD & 14.0 \\
\hline BAN/BRUT & 3.1 & BAN/DD & 11.6 \\
\hline CAN/BRUT & 2.7 & CAN/DD & 11.4 \\
\hline SAN/BRUT & 3.5 & SAN/DD & 13.1 \\
\hline STM/BRUT & 17.2 & STM/DD & 16.3 \\
\hline VPM/BRUT & 13.7 & VPM/DD & - \\
\hline AP/BRUT & - & BAF/BRUT & \\
\hline
\end{tabular}

Compte tenu de ces résultats, les limites d'Atterberg réalisées sur les sédiments bruts ont confirmé selon la classification des sols fins (abaque de plasticité de Casagrande), que les sédiments étudiés étaient des limons (silts) organiques plus ou moins plastiques (figure 3). Aussi un groupe de sédiments apparaît formé des sédiments référencés ARS, BAN, CAN et SAN.

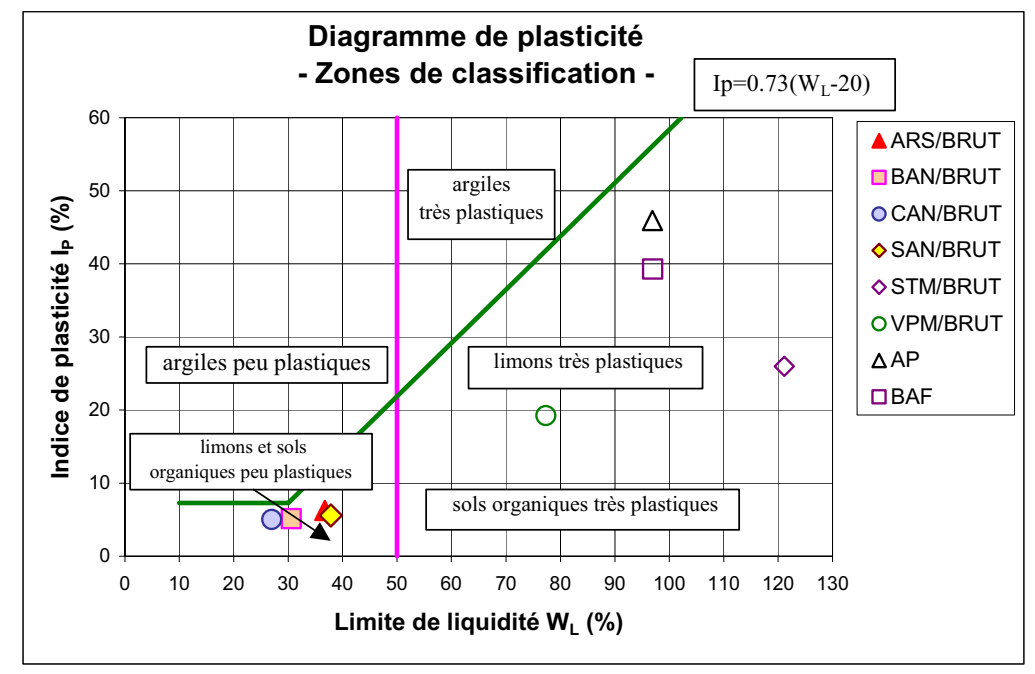

Figure 3. Classification des sédiments fins

\section{Paramètres hydrauliques}

Les propriétés hydrauliques font appel aux techniques usuelles utilisées pour les études de sédimentation et de consolidation de sols fins saturés. Ces propriétés fournissent les paramètres nécessaires aux calculs de tassement pour la mise en remblai des sédiments traités ou non (relation entre la perméabilité $\mathrm{k}$ et la concentration en matières solides $\mathrm{C}$ ). Les essais de décantation sont effectués pour 
des concentrations différentes et représentatives des sédiments prélevés selon les modes opératoires de dragage.

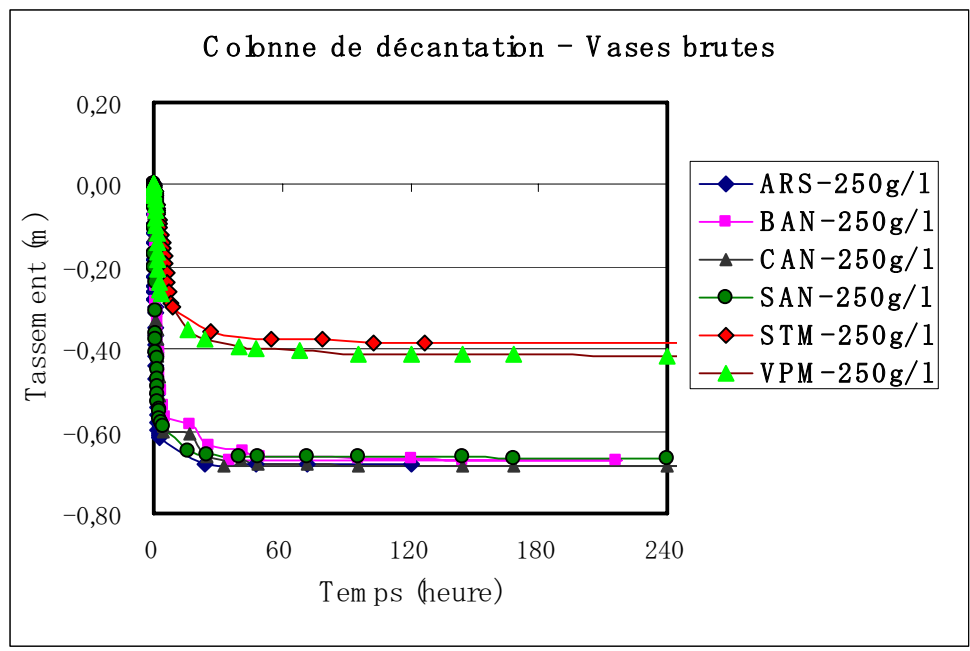

Figure 4. Tassement en colonne des sédiments bruts méditerranéens

Pour le cas des sédiments méditerranéens et une concentration à $250 \mathrm{~g} / \mathrm{l}$, le groupe de sédiments cité plus haut, a un même comportement en décantation, voir figure 4 alors que les deux autres plus organiques présentent un autre comportement. Pour ces mêmes sédiments et des concentrations initiales de $50 \mathrm{~g} / 1$ et $250 \mathrm{~g} / 1$, les essais à la colonne ont permis de déterminer l'évolution du coefficient de perméabilité $\mathrm{k}$ en fonction de la concentration $\mathrm{C}$. La relation entre $\mathrm{k}$ et $\mathrm{C}$ illustre aussi cette distinction (figure 5).

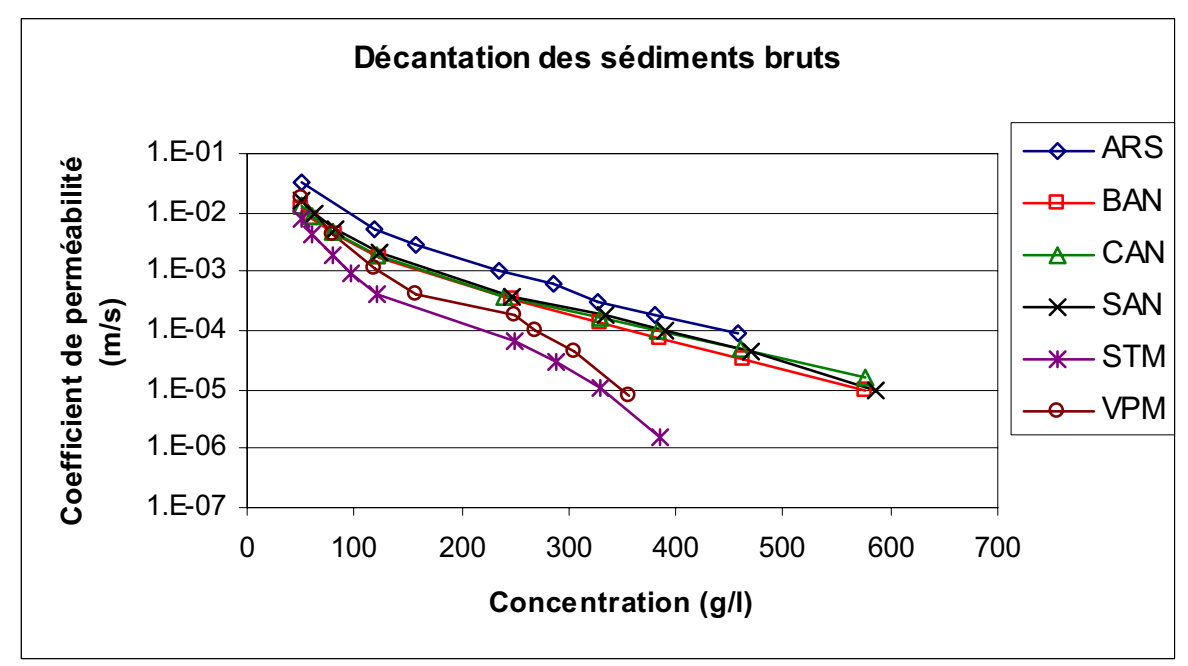

Figure 5. Relation k - C pour les sédiments bruts méditerranéens 


\section{Paramètres de consolidation}

Les essais œdométriques ont fourni les paramètres de tassement (coefficient de compression, coefficient de consolidation, coefficient de fluage) et permis de compléter la relation $\mathrm{k}-\mathrm{C}$ qui peut être proposée en fonction de l'indice des vides e. Ainsi, on peut voir sur la figure 6 l'évolution du coefficient de perméabilité $\mathrm{k}$ pour l'ensemble des essais de décantation (indice des vides élevé) et de consolidation et la continuité de cette évolution.

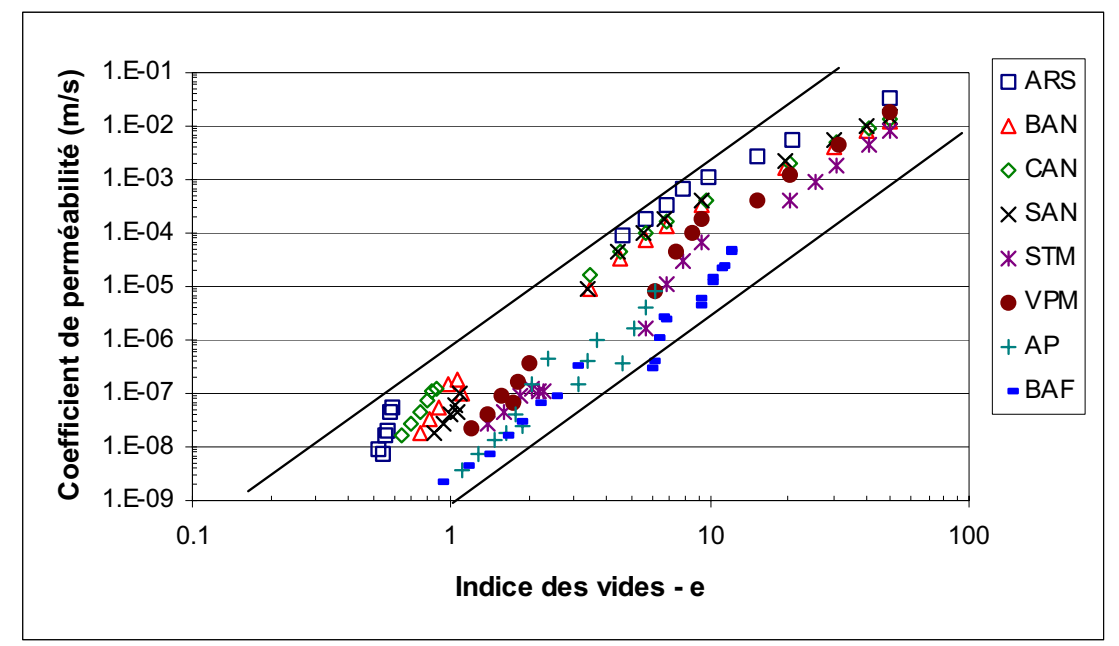

Figure 6. Relation k - e des sédiments bruts

Un autre résultat d'importance est relatif à l'évolution du coefficient de fluage $\mathrm{C} \alpha$ en fonction de la teneur des matières organiques (figure 7), la présence des matières organiques à teneur élevée dans les sédiments pose problème pour la mise en remblai sans traitement. D'une part, la détermination de la teneur en matières organiques sur les sédiments s'avère difficile même en respectant le protocole d'essai en vigueur (précision sur la mesure) et d'autre part, la variabilité des taux de matières organiques au sein même des sédiments prélevés existe et la représentativité de la mesure est parfois sujette à discussion (LIANG, 2008). Cependant un nombre répété de mesures permet d'avoir une valeur moyenne représentative. L'augmentation des teneurs des matières organiques tend à accroître le fluage comme on le voit sur la figure 7 pour les sédiments méditerranéens. La caractérisation physico mécanique des huit sédiments étudiés a apporté l'ensemble des paramètres nécessaires à toute valorisation en tant que matériau de remblai sans traitement en milieu confiné ou non selon le comportement environnemental. Il a été mis en lumière que quatre des six sédiments méditerranéens ont aussi un même comportement en fluage, ce résultat est très utile pour une caractérisation complémentaire en tant que matériau routier. 


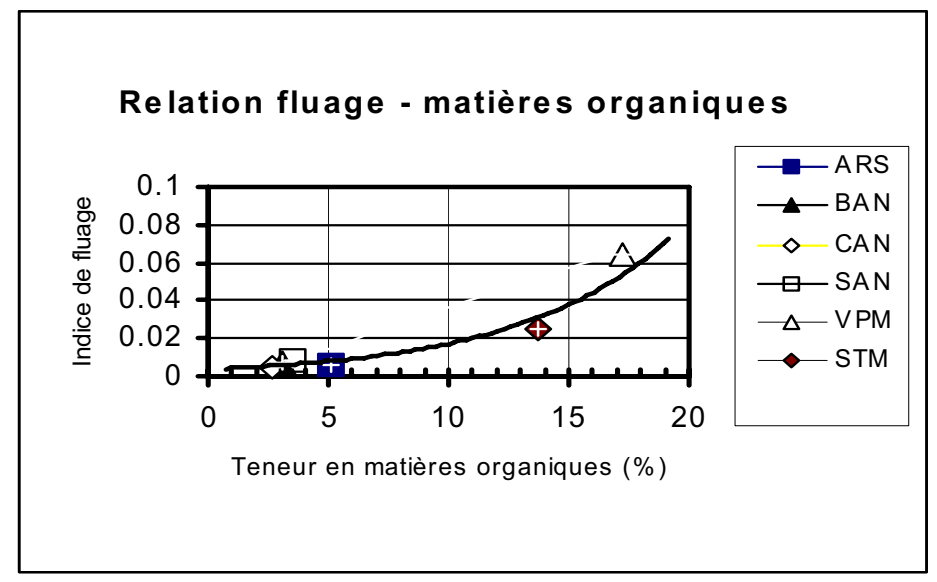

Figure 7. Relation indice de fluage $\boldsymbol{C}_{\alpha}$ et teneur en matières organiques des sédiments bruts

Le tableau 4 récapitule un ensemble de données relatives à la consolidation des sédiments marins. Des intervalles de valeurs communément admises (SETRA, 2000) pour des sols compressibles voire très compressibles permettent de situer les sédiments étudiés soit en fonction de leur caractère très organique ou de leur nature très argileuse. Ces données sur les sédiments sont précieuses pour le praticien.

Tableau 4. Propriétés hydrauliques et de consolidation des sédiments étudiés

\begin{tabular}{|c|c|c|c|c|c|c|}
\hline \multicolumn{7}{|c|}{ PROPRIETES CARACTERISTIQUES DES SEDIMENTS ETUDIES } \\
\hline PROPRIÉTÉS & $\begin{array}{l}\text { Teneur } \\
\text { en eau } \\
\text { w (\%) }\end{array}$ & $\begin{array}{c}\text { Indice } \\
\text { des vides } \\
\text { initial } \\
\mathbf{e}_{0} \\
\end{array}$ & $\begin{array}{c}\text { Compressibilité } \\
\mathrm{C}_{\mathrm{c}} /\left(1+\mathbf{e}_{0}\right)\end{array}$ & $\begin{array}{c}\text { Indice } \\
\text { de } \\
\text { fluage } \\
\mathrm{C}_{a} \\
\end{array}$ & $\begin{array}{c}\text { Coefficient } \\
\text { de } \\
\text { perméabilité } \\
\mathbf{k}(\mathrm{m} / \mathrm{s})\end{array}$ & $\begin{array}{c}\text { Coefficient } \\
\text { de } \\
\text { consolidation } \\
\mathrm{Cv}\left(\mathbf{m}^{2} / \mathbf{s}\right) \\
\end{array}$ \\
\hline Sédiment ARS & \multirow{6}{*}{$50-200$} & 0.593 & 0.030 & $0.133 \mathrm{C}_{\mathrm{c}}$ & \multirow{8}{*}{$10^{-6}$ à $10^{-9}$} & \multirow{6}{*}{$10^{-7}$ à $10^{-8}$} \\
\hline Sédiment BAN & & 1.113 & 0.117 & $0.027 \mathrm{C}_{\mathrm{c}}$ & & \\
\hline Sédiment CAN & & 0.899 & 0.091 & $0.028 \mathrm{C}_{\mathrm{c}}$ & & \\
\hline Sédiment SAN & & 1.102 & 0.082 & $0.046 \mathrm{C}_{\mathrm{c}}$ & & \\
\hline Sédiment STM & & 2.332 & 0.206 & $0.094 \mathrm{C}_{\mathrm{c}}$ & & \\
\hline Sédiment VPM & & 2.142 & 0.169 & $0.047 \mathrm{C}_{\mathrm{c}}$ & & \\
\hline Sédiment AP & \multirow{2}{*}{$80-200$} & 2.613 & 0.141 & - & & \multirow{2}{*}{$10^{-8}$ à $10^{-9}$} \\
\hline Sédiment BAF & & 3.424 & 0.121 & - & & \\
\hline \multicolumn{7}{|c|}{ PROPRIETES CARACTERISTIQUES DE SOLS COMPRESSIBLES* } \\
\hline Sols organiques & $100-200$ & 2 à 3 & 0.2 à 0.35 & \multirow{3}{*}{$\begin{array}{c}0.03 \\
\text { à } \\
0.05 \mathrm{C}_{\mathrm{c}}\end{array}$} & $10^{-6}$ à $10^{-9}$ & $10^{-6}$ à $10^{-8}$ \\
\hline Vases & $60-150$ & 1.5 à 3 & 0.25 à 0.4 & & $10^{-7}$ à $10^{-9}$ & $10^{-7}$ à $10^{-8}$ \\
\hline Argiles molles & $30-100$ & 1.2 à 2 & 0.15 à 0.3 & & $10^{-9}$ à $10^{-11}$ & $10^{-7}$ à $10^{-9}$ \\
\hline
\end{tabular}

Nota: * Propriétés résumées dans le guide technique "Etude et réalisation des remblais sur sols compressibles", SETRA-LCPC (2000). 


\section{Conclusions}

La méthodologie proposée qui est basée sur des essais utilisés en mécanique des sols et sur des essais de sédimentation permet d'obtenir un ensemble de données très utiles pour étudier le comportement de sédiments marins mis en remblai sans traitement préalable. Cependant pour une mise en remblai avec traitement de stabilisation, ces données sont aussi nécessaires ne serait-ce que pour établir des comparaisons dans les comportements. De ce fait, il s'agit d'une caractérisation minimale de sédiments avant toute valorisation en remblai ou équivalente.

Un autre intérêt est de constituer une base de données sur les propriétés des sédiments, soit sous forme de tableau, voir tableau 3, soit à partir de relations perméabilité - indice des vides, voir figure 6 ou soit sous forme de courbes modélisées reliant la perméabilité à la concentration des sédiments (SANCHEZ \& LEVACHER, 2007, DUAN, 2008), de type

$\mathrm{k}=\mathrm{A}_{1} \exp \left[-\mathrm{A}_{2}\left(\mathrm{C} / \rho_{\mathrm{s}}\right)^{0.65}\right]$

où $\mathrm{C}$ représente la concentration du sédiment, $\rho_{\mathrm{s}}$ la masse volumique de sa phase solide et $\mathrm{A}_{1}$ et $\mathrm{A}_{2}$ sont des paramètres propres à chaque sédiment. Ceci est illustré à la figure 8 .

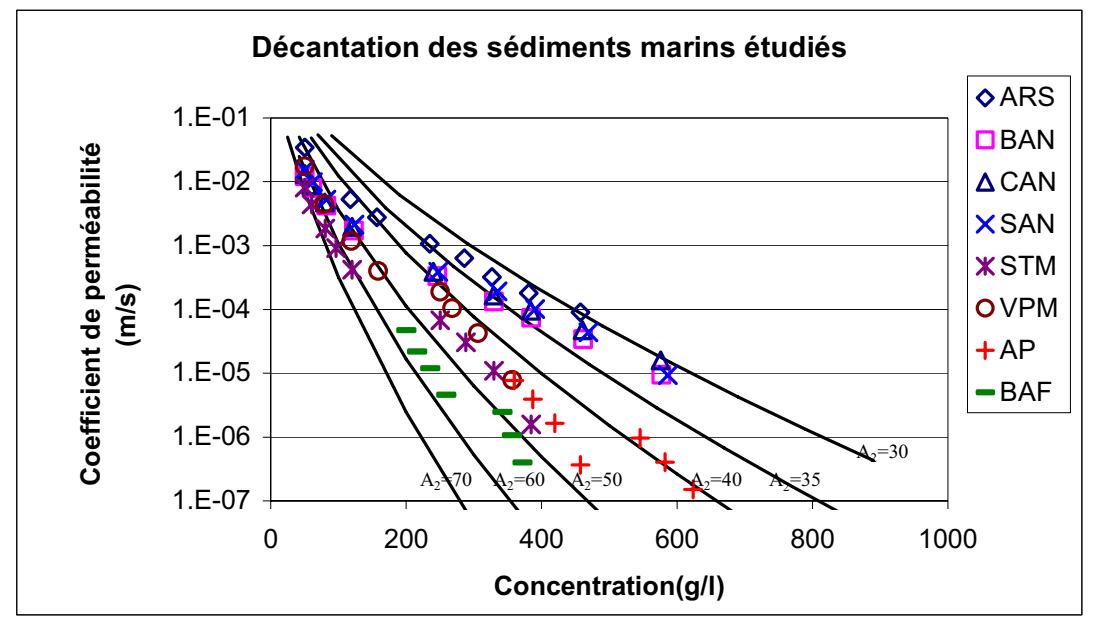

Figure 8. Evolution de $k$ en fonction de C pour les sédiments étudiés

\section{Références bibliographiques}

${ }^{1}$ ARRETE ministériel du 14 juin 2000 (2000). Valeurs de référence pour quelques contaminants métalliques et organiques contenus dans les sédiments marins ou estuariens. Circulaire ${ }^{\circ} 2000-62$, Journal Officiel $n^{\circ} 184 \mathrm{du}$ 10/08/00. pp 12415-12416. 
${ }^{2}$ COLIN D. (2003). Valorisation de sédiments fins de dragage en technique routière. Thèse de l'Université de Caen-Basse-Normandie, $181 \mathrm{p}$.

${ }^{3}$ DUAN Z. (2008) Caractérisation, stabilisation et solidification de sédiments marins. Thèse de l'Université de Caen-Basse-Normandie, 198 p.

4 DUBOIS V. (2006). Etude du comportement physico-mécanique et caractérisation environnementale des sédiments marins - valorisation en technique routière. Thèse de l'Ecole Nationale Supérieure des Mines de Douai et de l'Université d'Artois. 274 p.

5 LE GUERN C., CONIL P., CLOZEL-LELOUP B., LEVACHER D., SCHWARTZ C., PROULHAC N., ALBRECHT M., BATICLE P. (2004). Aide à la gestion alternative au rejet en mer de sédiments contaminés provenant $d u$ dragage de sites portuaires. Programme Liteau, Rapport final BRGM/RP-53470FR, $239 \mathrm{p}$.

${ }^{6}$ LEMEE F. (2006). Traitement par alcali activation de sédiments fins marins non contaminés et à faible teneur en eau - Mise au point d'un procédé de stabilisation. Thèse de l'Université de Caen-Basse-Normandie, $187 \mathrm{p}$.

${ }^{7}$ LEVACHER D., DUAN Z., LIU Z., SANCHEZ M. (2007a). Caractérisation mécanique de sédiments de ports méditerranéens en vue de leur réutilisation en remblai, Rapport d'avancement $\mathrm{n}^{\circ} 2$, Réf. $\mathrm{N}^{\circ} \mathrm{CFL}-07-\mathrm{CG83-01,} \mathrm{Centre} \mathrm{Français}$ du Littoral, 64 p.

8 LEVACHER D., DUAN Z., SANCHEZ M. (2007b). Caractérisation mécanique de sédiments de ports méditerranéens stabilisés, Rapport d'avancement $\mathrm{N}^{\circ}$ 2, Réf. $\mathrm{N}^{\circ} \mathrm{CFL}-07-\mathrm{CG} 83-02$, Centre Français du Littoral, $40 \mathrm{p}$.

${ }^{9}$ LEVACHER D., SANCHEZ M., DUAN Z., JIGOREL A., PROULHAC N. (2005). Etudes physico-mécaniques des matériaux de dragage du port de Cherbourg en vue d'une réutilisation en remblais. Rapport IDRA Rennes, $135 \mathrm{p}$.

${ }^{10}$ LIANG Y. (2008). Traitement de sédiments marins : amélioration de la résistance par des procédés de stabilisation/solidification par voie chimique et liants hydrauliques. Rapport Master 2 Génie Côtier, Université de Caen-BasseNormandie, 60 p. (à paraître, septembre 2008).

${ }^{11}$ SANCHEZ M., LEVACHER D. (2007). The influence of particle size of the dispersed mineral fraction on the settlement of marine and estuarine muds, Geomarine Letters, Ed. Springer, A21038, Volume 27, n 5, pp 303-313.

12 SEMCHA A. (2006). Valorisation des sédiments de dragage - Application dans le BTP - Cas de barrages algériens, Thèse de l'Université de Reims Champagne Ardenne, $173 \mathrm{p}$.

${ }^{13 .}$ SETRA-LCPC (2000). Etude et réalisation des remblais sur sols compressibles, Guide technique LCPC-SETRA, 85p. 
Ouvrages portuaires et gestion des sédiments 